\title{
Energy integral method for gravity field determination from satellite orbit coordinates
}

\author{
P.N.A.M. Visser ${ }^{1}$, N. Sneeuw ${ }^{2}$, C. Gerlach ${ }^{3}$ \\ ${ }^{1}$ Delft Institute for Earth-Oriented Space Research, Delft University of Technology, Kluyverweg 1, 2629 HS, Delft, The Netherlands \\ e-mail: pieter.visser@1r.tudelft.nl; Tel.: +31-15-2782595, Fax: +31-15-2785322 \\ ${ }^{2}$ Department of Geomatics Engineering, University of Calgary, 2500 University Drive, Calgary, Alberta, Canada T2N 1N4 \\ e-mail: sneeuw@ucalgary.ca; Tel.: +1-403-220-4703, Fax: +1-403-284-1980 \\ ${ }^{3}$ Institut für Astronomische und Physikalische Geodäsie Arcisstrasse 21, 80333 Munich, Germany \\ e-mail: gerlach@bv.tum.de; Tel.: +49-89-289-23179, Fax: +49-89-289-23178
}

Received: 23 January 2002 / Accepted 20 December 2002

\begin{abstract}
A fast iterative method for gravity field determination from low Earth satellite orbit coordinates has been developed and implemented successfully. The method is based on energy conservation and avoids problems related to orbit dynamics and initial state. In addition, the particular geometry of a repeat orbit is exploited by using a very efficient iterative estimation scheme, in which a set of normal equations is approximated by a sparse block-diagonal equivalent. Recovery experiments for spherical harmonic gravity field models up to degree and order 80 and 120 were conducted based on a 29-day simulated data set of orbit coordinates. The method was found to be very flexible and could be easily adapted to include observations of non-conservative accelerations, such as (to be) provided by satellites like CHAMP, GRACE, and GOCE. A serious drawback of the method is its large sensitivity to satellite velocity errors. Existing orbit determination strategies need to be altered or augmented to include algorithms that focus on optimizing the accuracy of estimated velocities.
\end{abstract}

Keywords: Energy integral - Gravity field determination - Accelerometer observations Block-diagonal matrix - Orbit errors Orbit coordinates

\section{Introduction}

Satellite tracking observations have been used successfully in global gravity field modeling over the last two decades. However, no satellites were launched with the primary aim of mapping the Earth's gravity field before July 2000, when CHAMP was put into orbit (Reigber et al. 1999). The US/German GRACE and European Space Agency (ESA) GOCE satellites will follow in 2002 and 2006, respectively (ESA 1999; Tapley and Reigber

Correspondence to: P.N.A.M. Visser
1999). All these satellites are and will be equipped with high-quality space-borne receivers that will allow highlow satellite-to-satellite tracking ( $h-\ell$ SST) of the US Global positioning system (GPS). SST observations provide accurate information for recovering the orbits of satellites, which are primarily determined by the Earth's gravity field. Non-conservative forces, as induced by atmospheric drag, solar radiation and possibly attitude and orbit control systems, will be measured by on-board accelerometers, preventing the aliasing of these forces in the gravity field solutions. Moreover, high-resolution gravity field recovery will be enhanced by low-low SST (1-1 SST) for GRACE and satellite gravity gradiometry (SGG) for GOCE. For all three missions, the objectives cannot be met without the acquisition of GPS SST observations. GPS SST observations of low Earth-orbiting satellites have been employed successfully in gravity field models such as EGM96 (Lemoine et al. 1997) and GRIM5-S1 (Biancale et al. 2000) where use was made of classical numerical integration techniques in establishing the observation equations, which are in general solved by L2-norm techniques resulting in normal equation systems. The most common representation of the Earth's gravity field in satellite geodesy is a series expansion into spherical harmonic functions. For high-degree and -order spherical harmonic expansions, the size of the normal equations becomes very large, posing strong demands on computer memory. In addition, the computation of full sets of normal equations for large spherical harmonic expansions requires extensive computer resources. For missions such as CHAMP, GRACE, and GOCE, it is foreseen that spherical harmonic expansions are required to at least degree and order 100, 150, and 300 , or 10000,22500 , and 90000 gravity field coefficients, respectively, for the full complement of observations that will be provided by the respective satellites (ESA 1999). Considering GPS SST observations only, it can be shown that for especially the very low flying GOCE satellite (about $250 \mathrm{~km}$ altitude) gravity field terms up to at least degree and order 150 are relevant SID (2000). For CHAMP and GRACE the 
modeling and estimation of gravity field terms above degree 100 are also required (Reigber et al. 2001). Extensive computer resources are required to compute such normal equation systems directly and store them in memory.

A computationally efficient alternative method is proposed that consists of two steps. First, a high-precision orbit is computed from the GPS observations using, for example, a kinematic or reduced-dynamic orbit determination technique (Visser and van den IJssel 2000). Second, the resulting position and velocity coordinates are used as observations for gravity field recovery. The focus of this paper will be on the second step. The gravity field recovery is formulated in such a way that orbit unknowns, such as the epoch state vector (start position and velocity), are eliminated entirely, in addition to linearization errors which are included when using conventional techniques. This way, problems that might arise due to correlations between unknown gravity field parameters and state vector unknowns, and due to linearization errors, are prevented (Visser et al. 2001).

The formulation is based on energy integrals, such as proposed and described by Jekeli (1999), Reigber (1969), Ilk (1983), and O'keefe (1957). An efficient iterative method, in the following referred to as iterative energy integral method, for gravity field recovery from orbit coordinates based on energy considerations and blockdiagonal dominance of normal equation systems will be outlined in detail. This method has been implemented successfully and different gravity field recovery experiments will be discussed, complete to degree and order either 80 or 120 . The gravity recovery error has been found to be negligible. Attention will be paid to incorporation of dissipative accelerations in the energy integrals. These accelerations may, for example, be measured by on-board accelerometers. Finally, an assessment has been made of the sensitivity of the method to orbit errors.

\section{Method}

First, the observation equations for the gravity field recovery from orbit perturbations are established. This is followed by a description of the iterative solution strategy.

\subsection{Observation equations}

Conventional methods for gravity field recovery from satellite tracking observations rely on numerical integration techniques in order to solve the variational equations (Montenbruck and Gill 2000). The solution of these variational equations is required to establish the observation equations. The method proposed here consists of two steps: (1) a precise orbit determination and (2) gravity field recovery using the orbit solution. The orbit estimation is thus decoupled from the gravity field recovery. It is assumed that the GPS receiver provides a continuous data stream of $\mathrm{h}-1 \mathrm{SST}$ observations allowing the accurate determination of a continuous time series of satellite positions and velocities, e.g. by a kinematic or reduced-dynamic technique (Visser and van den IJssel 2000). The orbit solution can thus be seen as a condensed set of GPS tracking observations. Conceptually, it is assumed that this solution is free of dynamic model errors.

The gravity recovery will be based on a least-squares (LS) solution of observation equations linking the orbit position and velocity solution to the gravity field unknowns. The gravity field potential $U$ is represented by a spherical harmonic expansion

$$
\begin{aligned}
U=\frac{\mu}{r}\{1 & +\sum_{l=2}^{\infty} \sum_{m=0}^{l}\left(\frac{a_{e}}{r}\right)^{l}\left(\bar{C}_{l m} \cos m \lambda\right. \\
& \left.\left.+\bar{S}_{l m} \sin m \lambda\right) \bar{P}_{l m}(\sin \phi)\right\}
\end{aligned}
$$

where $\mu$ is the gravity parameter, $a_{e}$ is the mean equatorial radius, $r, \phi, \lambda$ are the spherical coordinates (radius, geocentric latitude, longitude), $\bar{P}_{l m}$ is the normalized Legendre polynomial of degree $l$ and order $m$, and $\bar{C}_{l m}, \bar{S}_{l m}$ represent the (unknown) gravity parameters.

The solved positions and velocities will not be used directly, but will be inserted in an energy integral in order to establish the observation equations for the gravity recovery. The reference frames that are particularly relevant for describing Earth satellite motion and the Earth gravity potential are the Earth-centered, inertial reference frame (ECI) and the Earth-centered, Earth-fixed reference frame (ECEF). In fact, the ECI frame is a pseudo-inertial reference frame due to the influence of third bodies such as the Sun, Moon, and planets, but for the moment this influence is not taken into consideration and the real world is considered to consist of a uniformly rotating Earth with angular rotation rate $\bar{\omega}$. The starting point is the equation for the Lagrangian $L$ in the inertial ECI frame, which can be written as

$L=T-V=\frac{1}{2} \bar{v}_{i} \cdot \bar{v}_{i}-V$

where $T$ is the kinetic energy per unit of mass and $V$ the potential energy per unit of mass in the physics sense (in the remainder of this paper the simple term 'energy' will be used instead of 'energy per unit of mass'). The sign of the potential energy $V$ in the physics sense is opposite to the sign of the potential $U$ [Eq. (1)] in the geodetic sense $(V=-U)$. The velocity vector in the ECI frame is represented by $\bar{v}_{i}$. For the ECEF frame, the following relation can be derived:

$$
\begin{aligned}
\bar{v}_{i} & =\bar{v}_{e}+\bar{\omega} \times \bar{r}_{e} \\
L & =\frac{1}{2} \bar{v}_{e} \cdot \bar{v}_{e}+\bar{v}_{e} \cdot\left(\bar{\omega} \times \bar{r}_{e}\right)+\frac{1}{2}\left(\bar{\omega} \times \bar{r}_{e}\right) \cdot\left(\bar{\omega} \times \bar{r}_{e}\right)-V
\end{aligned}
$$

where $\bar{r}_{e}$ and $\bar{v}_{e}$ are the position and velocity vector in the ECEF frame, respectively. The next step in deriving 
the final observation equation is the transformation from the Lagrangian to the Hamiltonian $H$

$H=\bar{p}_{e} \cdot \bar{v}_{e}-L$

where $\bar{p}_{e}$ is the vector with generalized momenta (Lanczos 1949; Landau and Lifschitz 1965)

$\bar{p}_{e}=\frac{\partial L}{\partial \bar{v}_{e}}=\bar{v}_{e}+\bar{\omega} \times \bar{r}_{e}$

Thus, Eq. (4) can be rewritten as

$H=\frac{1}{2} \bar{v}_{e} \cdot \bar{v}_{e}-\frac{1}{2}\left(\bar{\omega} \times \bar{r}_{e}\right) \cdot\left(\bar{\omega} \times \bar{r}_{e}\right)+V$

The part $\frac{1}{2}\left(\bar{\omega} \times \bar{r}_{e}\right) \cdot\left(\bar{\omega} \times \bar{r}_{e}\right)$ is the centrifugal potential. The static potential energy $V$ does not change in the ECEF frame. The Hamiltonian $H$ can be seen as the total energy of the underlying mechanical system and is a constant of the motion. By setting $\bar{v}_{e}=\bar{v}_{i}-\bar{\omega} \times \bar{r}_{e}$, we arrive at

$H=\frac{1}{2} \bar{v}_{i} \cdot \bar{v}_{i}-\bar{v}_{i} \cdot\left(\bar{\omega} \times \bar{r}_{e}\right)+V$

or by cyclical change of the triple vector product

$H=\frac{1}{2} \bar{v}_{i} \cdot \bar{v}_{i}-\bar{\omega} \cdot\left(\bar{r}_{e} \times \bar{v}_{i}\right)+V$

It can be clearly seen that a rotating potential requires an additional contribution to the simple Hamiltonian $H_{0}=\frac{1}{2} \bar{v}_{i} \cdot \bar{v}_{i}+V$, namely the angular momentum term $\bar{\omega} \cdot\left(\bar{r}_{e} \times \bar{v}_{i}\right)$ which appears as a kinetic energy term $\bar{v}_{i} \cdot \bar{v}_{k}$ when replacing $\bar{\omega} \times \bar{r}_{e}$ by $\bar{v}_{k}$.

The observation equation is now obtained by inserting the positions and velocities obtained by the precise orbit determination and Eq. (1) (with $V=-U$ ) in Eq. (8) and in addition using the principle of energy conservation stating that the Hamiltonian is constant. This constant may be considered as an additional unknown that needs to be estimated simultaneously with the unknown gravity field parameters. In the above derivation it is assumed that no dissipative accelerations are present. It is interesting to make a comparison with the formulation by Jekeli (1999). He presented an alternative derivation leading to the same result. However, he included a term taking into account dissipative accelerations. This term can be thought of as an energy loss $D$ and becomes equal to

$D=\int_{0}^{\tau} \bar{a}_{e} \cdot \bar{v}_{e} \mathrm{~d} t$

where $\bar{a}_{e}$ is the acceleration vector in the ECEF frame.

Based on the above, and assuming that the Earth is rotating with a constant rotation rate $\omega_{e}$ around the polar $z$-axis, the following equation can be derived from Eq. (6) using ECEF coordinates:

$H=-U\left(x_{e}, y_{e}, z_{e}\right)+\frac{1}{2}\left\{\dot{x}_{e}{ }^{2}+\dot{y}_{e}{ }^{2}+\dot{z}_{e}{ }^{2}\right\}-\frac{1}{2} \omega_{e}{ }^{2}\left\{x_{e}{ }^{2}+y_{e}{ }^{2}\right\}$ or, when making use of ECI coordinates for the kinetic energy and centrifugal terms (as coded in the used software)

$$
\begin{aligned}
H=-U\left(x_{e}, y_{e}, z_{e}\right)+\frac{1}{2}\left\{\left(\dot{x}_{i}+\omega_{e} y_{i}\right)^{2}\right. & \left.+\left(\dot{y}_{i}-\omega_{e} x_{i}\right)^{2}+\dot{z}_{i}^{2}\right\} \\
& -\frac{1}{2} \omega_{e}^{2}\left\{x_{i}^{2}+y_{i}^{2}\right\}
\end{aligned}
$$

where the Hamiltonian $H$ is constant in the case of no energy dissipation, $x_{e}, y_{e}, z_{e}$ and $\dot{x}_{e}, \dot{y}_{e}, \dot{z}_{e}$ are the Cartesian position and velocity coordinates in the ECEF frame; and $x_{i}, y_{i}, z_{i}$ and $\dot{x}_{i}, \dot{y}_{i}, \dot{z}_{i}$ are the Cartesian positions and velocities in the ECI frame.

Combining Eqs. (11) and (9) yields the observation equation for the gravity potential

$$
\begin{aligned}
H+U\left(x_{e}, y_{e}, z_{e}\right)=\frac{1}{2}\left\{\left(\dot{x}_{i}+\omega_{e} y_{i}\right)^{2}+\left(\dot{y}_{i}-\omega_{e} x_{i}\right)^{2}+\dot{z}_{i}^{2}\right\} \\
\\
-\frac{1}{2} \omega_{e}{ }^{2}\left\{x_{i}^{2}+y_{i}^{2}\right\}-\int_{0}^{\tau} \bar{a}_{e} \cdot \bar{v}_{e} \mathrm{~d} t .
\end{aligned}
$$

In this equation, the unknowns $H$ and $\bar{C}_{l m}, \bar{S}_{l m}$ are included in the left-hand side, and observed orbit components in the ECI and ECEF frames in the righthand side, in addition to possibly observed or modeled dissipative accelerations $\bar{a}_{e}$. In principle, Eq. (12) can be further extended to include additional terms such as those due to 3rd-body perturbations (Sun, Moon, and planets), and solid-Earth and ocean tides, either in the form of corrections (right-hand side) or unknowns (lefthand side). Including additional unknowns might have consequences for the iterative solution strategy: the gravity part of the normal equations will remain blockdiagonally dominant (assuming a continuous stream of orbit coordinate solutions for a repeat period; see Sect. 2.2), a feature that can still be exploited in solution strategies, but the parts of the normal matrix due to the additional unknowns might have a different structure. In addition, the method can be modified to include a more realistic motion of the Earth in inertial space (precession, nutation, polar motion). Also, nuisance parameters such as accelerometer biases and scale factors can be added as either corrections or unknowns. These inclusions and additions are, however, beyond the scope of this paper. The research described in this paper primarily served to test the concept of using energy integral methods for deriving gravity from orbit perturbations and did not aim at developing a comprehensive software package that can take into account all kinds of known physical models in addition to the Earth's static gravity field.

\subsection{Iterative solution strategy}

Equation (12) is linear with respect to the gravity unknowns. In the case of classical methods, the observation equations are not really linear but linearized. In 
other words, when using classical methods the observation equations are approximated by a first-order Taylor expansion. This means that higher-order terms are neglected and approximation errors are introduced, which sometimes requires more iterations depending on the quality of the starting values and the nonlinearity of the problem. Using Eq. (12), no approximation errors are introduced. In principle, the gravity recovery can be completed in one step, as opposed to the multiple iterations that are generally required in the classical approach. However, in order to solve the observation equations in one step a full matrix approach is required, while here a fast and numerically stable solution method is aimed at.

An iterative method is selected where the normal matrix is approximated by a block-diagonal matrix. It is required that the normal matrix is organized per order. The method was tested successfully for SGG observations and principles and implementation aspects are described in detail in Rummel and Colombo (1985) Klees et al. (2000), SID (2000), Visser et al. (2001). The method is particularly suitable for satellites that fly a near-circular repeat orbit and provide observations at a constant sampling rate. The potential $U$ can be represented as a series expansion in Kepler elements. Assuming a circular orbit with zero eccentricity $e$, the following relation can be derived (cf. Kaula 1966, Sect. 3.3):

$$
O=H+U=H+\frac{\mu}{a} \sum_{l=2}^{l_{\max }} \sum_{m=0}^{l} \sum_{p=0}^{l}\left(\frac{a_{e}}{a}\right)^{l} F_{l m k}(i) S_{m k}(u, \Lambda)
$$

$$
\begin{aligned}
S_{m k}(u, \Lambda)= & {\left[\begin{array}{c}
\bar{C}_{l m} \\
-\bar{S}_{l m}
\end{array}\right] \cos (k u+m \Lambda) } \\
& +\left[\begin{array}{c}
\bar{S}_{l m} \\
\bar{C}_{l m}
\end{array}\right]_{l-m \text { odd }}^{l-m \text { even }} \sin (k u+m \Lambda)
\end{aligned}
$$

where $O$ represents the right-hand side of Eq. (12) and includes the orbit solution, $l_{\max }$ is the maximum degree at which the series expansion is truncated, $k$ is equal to $l-2 p, u$ is equal to $\omega+M$, and $\Lambda$ to $\Omega-\theta$. Use has been made of the Kepler elements: the orbital semimajor axis $a$ (equal to the radius $r$ for a circular orbit), argument of perigee $\omega$, inclination $i$, right ascension of ascending node $\Omega$, and the mean anomaly $M$. The Greenwich mean sidereal time is denoted by $\theta$, while $F_{l m k}(i)$ is a function depending on the orbital inclination $i$ only. The argument of the cosine and sine components of the function $S_{m k}(u, \Lambda)$ can be written as

$$
\begin{aligned}
\psi & =k u+m \Lambda=f_{m k} \omega_{\text {sat }} t=\left(k-\frac{m}{n_{r}}\right) \omega_{\text {sat }} t \\
& =\left(k-m \frac{n_{\text {day }}}{n_{\text {rev }}}\right) \omega_{\text {sat }} t
\end{aligned}
$$

where $\omega_{\text {sat }}$ is the orbital angular velocity and $n_{r}$ is the number of revolutions per nodal day, which for a repeat orbit is the ratio of two relative primes $n_{\text {rev }}$ and $n_{\text {day }}$, where the repeat period is equal to $n_{\text {day }}$ nodal days in which the satellite completes $n_{\text {rev }}$ orbital revolutions. With a continuous data stream of observations $O$ and assuming a data set spanning a multiple integer of repeat orbits, the observations can be represented by a Fourier series and the normal matrix becomes block diagonal when the gravity unknowns are organized per order (Colombo 1984; Visser 1992). The normal equations can then be computed very fast and efficiently.

The exact observation equations [Eq. (12)] will be approximated by Eqs. (13) and (14) in a way similar to that described in Klees et al. (2000) and Visser et al. (2001), where the real orbit is approximated by a bestfitting circular repeat orbit. Visser et al. (2001) used the following notation for the exact observation equations and, in conjunction, normal equations:

$A_{t} \bar{x}=\bar{y}, N_{t} \bar{x}=A_{t}{ }^{T} A_{t} \bar{x}=A_{t}{ }^{T} \bar{y}$

and for the approximated observation and normal equations

$A_{a} \bar{x}=\bar{y}, N_{a} \bar{x}=A_{a}{ }^{T} A_{a} \bar{x}=A_{a}{ }^{T} \bar{y}$

where $A_{t}$ and $A_{a}$ are the design matrices, $\bar{x}$ represents the vector of unknowns $H, \bar{C}_{l m}, \bar{S}_{l m}$, and $\bar{y}$ the vector of observations $O$. Then the model or approximation error $\Delta \bar{x}$ becomes

$\Delta \bar{x}=\left(N_{t}^{-1} A_{t}-N_{a}^{-1} A_{a}\right) \bar{y}$

In Klees et al. (2000) an iterative scheme has been adopted to eliminate the approximation errors, which will also be used to solve the observation equations based on Eq. (12). Applying this scheme results in a recomputation after each iteration of the right-hand side of the normal equations using the same $A_{a}$ matrix, but with updated observations $O$. The left-hand side remains constant, i.e. the block-diagonal matrix $N_{a}$. After some manipulations Klees et al. (2000), it can be shown that the iterative process only converges to the right solution when the following criterion is met:

$\rho\left(I-N_{a}^{-1} A^{T}{ }_{a} A_{t}\right)<1$

where $\rho$ is the spectral radius and $I$ the identity matrix. The spectral radius of a matrix is defined as its maximum eigenvalue [in Eq. (19) of $\left.I-N_{a}{ }^{-1} A^{T}{ }_{a} A_{t}\right]$. If $A_{a}$ is a close approximation of $A_{t}$, the following formula will give a good indication for convergence (Visser et al. 2001):

$\rho\left(I-\left[N_{t}\right]_{b d}{ }^{-1} N_{t}\right)<1$

where $\left[N_{t}\right]_{b d}$ is the block-diagonal part of the true normal matrix. Actual computations of the spectral radius will be discussed in Sect. 4.1.

\section{Configuration}

The satellite orbital position and velocity in the ECEF frame form the basic observables for the energy integral equation [Eq. (10)]. In order to assess the feasibility of 
the iterative energy integral method, a time series of these positions and velocities was generated assuming a pure gravitational orbit, i.e. the orbit was determined by a static gravity field model only. Moreover, the Earth was modeled as rotating around the polar axis with constant angular velocity. The resulting equations of motion were solved by applying a 10th-order Adams-Moulton integrator (Boyce and DiPrima 1986). A GOCE-type orbit was selected, which repeats to within a few kilometers after 29 days, in which the satellite completes 467 orbital revolutions. The average altitude is equal to $250 \mathrm{~km}$. The inclination is equal to $96^{\circ}$, leading to a sunsynchronous orbit. Such an orbit will be sensitive to gravity field terms up to at least degree 100, and significant gravity field terms might be recovered beyond this degree (Colombo 1984; Visser et al. 1994; ESA 1998). Therefore, the Earth's gravity field was modeled by a spherical harmonic expansion complete to degree and order 120. This truncation degree was adopted in both the nominal orbit integration and gravity recovery, enabling a closed-loop test of the iterative energy integral method (in some cases the maximum degree was equal to 80, as will be explained in Sect. 4). The EGM-96 gravity field model was used to represent the real world (truncated at the appropriate degree).

The resolution of a $120 \times 120$ gravity field model is equal to about $175 \mathrm{~km}$ at $250 \mathrm{~km}$ altitude. It takes the satellite about $22 \mathrm{~s}$ to traverse such a distance and the Adams-Moulton orbit integration step has to be smaller. In the recovery experiments it was taken equal to $10 \mathrm{~s}$, equal to the time interval of the observed orbital positions and velocities.

An important aspect will be the inclusion of accelerometer observations in the iterative energy integral method. These observations will provide a measure of the dissipative forces that act on the satellite. CHAMP, GRACE, and GOCE are or will be equipped with accelerometers providing these observations. In addition, GOCE will be equipped with a drag-free control (DFC) system that will reduce the non-conservative accelerations below the level of $10^{-6} \mathrm{~m} / \mathrm{s}^{2}$. The remaining accelerations will be measured with very high accuracy, also at low frequencies (ESA 1999). Because a GOCEtype orbit was selected for the simulations, it is assumed that the satellite will experience random accelerations with this magnitude that are measured by the accelerometers. In the recovery experiment, these accelerations are included both in the orbit integration, by generating a random time series with a Gaussian distribution, and as observations in the gravity field recovery. The time step of this time series was taken equal to $10 \mathrm{~s}$, i.e. equal to the time step of the Adams-Moulton orbit integration.

\section{Results}

The gravity recovery experiments were all based on the selected 29-day repeat period with the gravity field model complete to degree and order 80 or 120 . The experiments included the computation of spectral radii, iterative gravity solutions, incorporation of accelerometer observations and orbit error sensitivity analyses.

\subsection{Spectral radii computations}

First, the spectral radius according to Eq. (20) has been computed as a function of the maximum degree of the gravity field model recovery. A comparison has been made with spectral radii obtained for gravity field recovery from other observables using the same orbit geometry, namely $\mathrm{h}-1$ SST and SGG observables. The $\mathrm{h}-1$ SST observations and the associated normal equations were obtained by numerical integration of the observation equations using the GEODYN software (Rowlands et al. 1995). The SGG observations and associated normal equations were computed with similar procedures and software as outlined in Klees et al. (2000). The spectral radii are displayed in Fig. 1 up to degree 80 [for the SGG case they are an extension of the spectral radii displayed in Visser et al. (2001, Fig. 4)]. It can be seen that the iterative energy integral method seems feasible: the spectral radius is well below 1 up to degree and order 80 and does not seem inclined to increase rapidly for higher degrees. In fact, the spectral radius seems to 'compete' with the spectral radius for the SGG observables. It can also be seen that a blockdiagonal iterative approach based on classical numerical integration methods for $\mathrm{h}-1$ SST will not be feasible, as already found by Visser et al (2001).

\subsection{Gravity recovery experiment}

Encouraged by the spectral radii computations, a gravity field recovery experiment was conducted where the EGM-96 model complete to degree and order 120 was used to simulate a 29-day time series of positions and velocities. The iterative energy integral method was

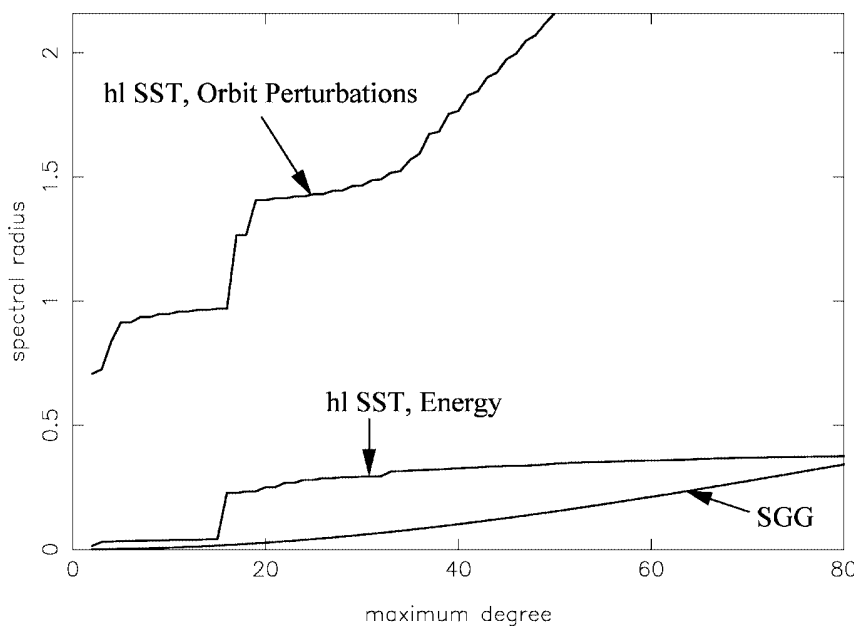

Fig. 1. Spectral radii for iterative solution schemes based on approximation of the full normal matrix by a reduced block-diagonal normal matrix: orbit perturbations derived from SST observations (h1 SST), energy equation derived from orbit perturbations (h-1 SST), and space-borne gravity gradients (SGG) 
used to recover all gravity coefficients from degree 2 to degree 120 , where the a priori values were set to zero. Thus the entire gravity signal was to be recovered, apart from the central term. It was found that the solution converged after seven iterations (Fig. 2), where convergence was defined to be achieved when the total gravity field adjustment in a certain iteration was smaller than $1 \mathrm{~mm}$ in terms of global geoid root mean square (RMS). After convergence, one more iteration is always made as an additional check of convergence. It is interesting to note that, before convergence, gravity recovery errors concentrate at resonance errors, i.e. at order zero and multiples of 16 , which is close to the number of orbit revolutions per day. In addition, the convergence of zonal gravity field terms is relatively slow. This can be explained by the fact that the full normal matrix displays clear side bands at the resonance orders, cf. Visser et al. (2001), which are not represented in the block-diagonal matrix which is used in the iterative solution procedure. Obviously, the recovery error due to approximating the full matrix by a block-diagonal matrix is eliminated by iteration, which can be explained by the fact that the spectral radius $\rho$ [Eq. (19)] is smaller than 1. The gravity field recovery error is found to be equal to $9 \mathrm{~mm}$ after convergence in terms of global geoid RMS when the zonal terms are excluded. Including these terms, the global geoid RMS error fluctuates between 2 and $7 \mathrm{~cm}$. This fluctuation can be attributed to instabilities in the normal equations due to the orbital inclination of $96^{\circ}$, leading to the well-known polar gap phenomenon (Visser et al. 1994; Sneeuw and Van Gelderen 1997; ESA 1999), that primarily affect low-order, and thus also zonal, gravity field terms. Regularization schemes can be applied, and are in fact required when going to higher degrees and orders. The subject of regularization is, however, beyond the scope of this paper. It was found
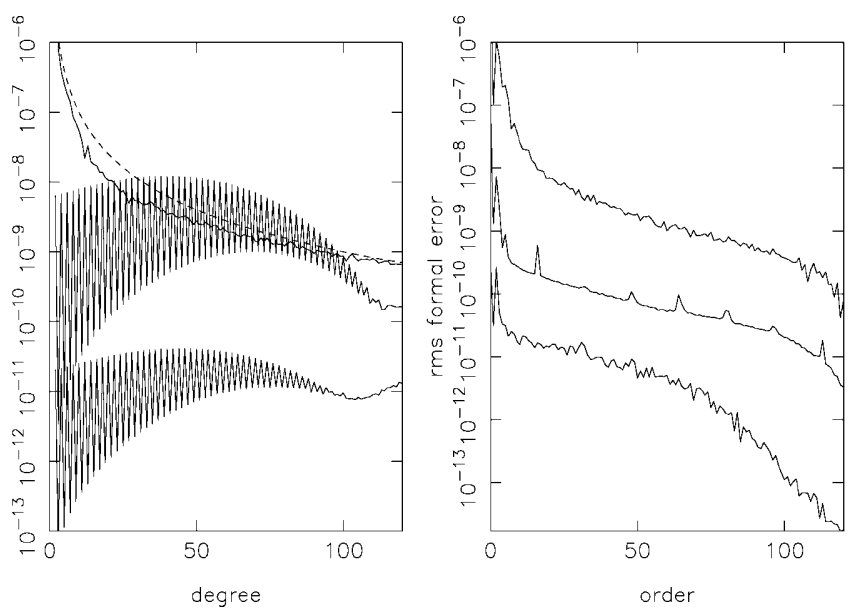

Fig. 2. Result of a gravity field recovery experiment complete to degree and order 120 based on a 29-day simulated data set of errorfree orbit perturbations. The RMS of the formal errors of the spherical harmonic expansion is displayed as a function of degree (left) and order (right). The dashed line represents Kaula's rule of thumb (left). The other three lines denote from top to bottom the total gravity signal and the recovery error after the 4th and the 7th iteration, respectively (left and right) that almost the entire geoid error is located in these polar gaps. For example, the global geoid error RMS, including the zonal terms, is equal to $20 \mathrm{~mm}$ after the 7 th iteration, whereas this error is equal to $5 \mathrm{~mm}$ when excluding the polar gaps, i.e. the areas south of $-84^{\circ}$ latitude and north of $84^{\circ}$ latitude (Table 1). After the 7 th iteration, only significant gravity recovery changes can be found in the polar gap areas (which represent less than $1 \%$ of the total Earth's surface).

The remaining 5- $\mathrm{mm}$ geoid error can be attributed for the larger part to energy dissipation, or equivalently to errors in the positions and velocities, due to very small, but significant, orbit integration errors. If the 10th-order Adams-Moulton was infinitely accurate, the sum of kinetic and potential energy along the computed orbit would be perfectly constant. However, it is found that this sum varies with an RMS about mean of 0.0018 $\mathrm{m}^{2} / \mathrm{s}^{2}$ in terms of energy. Using the inverse of the blockdiagonal approximation of the normal matrix scaled with this value, the predicted gravity field recovery error becomes as displayed in Fig. 3. The formal error is equal

Table 1. Gravity field recovery with the iterative energy integral method

\begin{tabular}{llll}
\hline $\begin{array}{l}\text { Maximum } \\
\text { degree }\end{array}$ & Geoid error $(\mathrm{mm})$ & $\begin{array}{l}\text { Observation error } \\
\text { Due to numerical. } \\
\text { integration }\left(\mathrm{m}^{2} / \mathrm{s}^{2}\right)\end{array}$ \\
\cline { 2 - 3 } & Global & No polar gaps & \\
\hline $120^{\mathrm{a}}$ & 20 & 5 & 0.0018 \\
$120^{\mathrm{b}}$ & 25 & 6 & 0.0027 \\
$80^{\mathrm{c}}$ & 4 & & 0.0058 \\
$80^{\mathrm{d}}$ & 585 & & 0.6226 \\
$80^{\mathrm{e}}$ & 585 & & 0.6229 \\
\hline
\end{tabular}

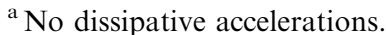

${ }^{\mathrm{b}}$ Random dissipative accelerations: $10^{-6} \mathrm{~m} / \mathrm{s}^{2}$.

${ }^{c}$ Orbit errors included: position errors only.

d 7Orbit errors included: velocity errors only.

e Orbit errors included: position + velocity errors.
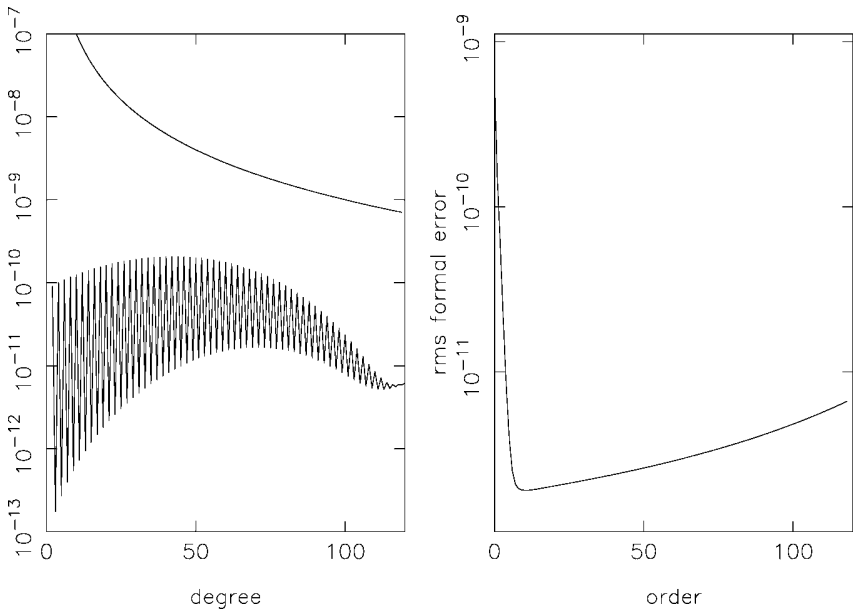

Fig. 3. Formal gravity field recovery error (cf. Fig. 2) obtained from the inverse of the block-diagonal normal approximation for a 29-day observation period. The formal errors were scaled according to the RMS about mean of the energy equation errors due to orbit integration error 
to $6.3 \mathrm{~cm}$ in terms of global geoid RMS, and $3 \mathrm{~mm}$ when excluding the polar gaps. The actual and prediced formal gravity recovery errors are in good agreement with each other. It can thus be concluded that the iterative gravity recovery scheme leads to a good solution, where remaining errors are largely caused by numerical integration imperfections.

\subsection{Dissipative accelerations}

The separation of conservative from non-conservative forces, mainly inflicted by atmospheric drag and solar radiation, but also for example by maneuvers and albedo, is crucial to the success of all gravity missions. This separation is (or will be) enabled by the provision of measurements of the non-conservative accelerations by space-borne accelerometers providing time series of such measurements.

Gravity field recovery experiments with the iterative energy integral method were conducted where the observation equations include corrections based on accelerometer observations, i.e. the integral term $\int_{0}^{\tau} \bar{a}_{e} \cdot \bar{v}_{e} \mathrm{~d} t$ [Eq. (12)] was taken into account. As with the numerical orbit integration, use was made of a 10th order AdamsMoulton integrator to evaluate the integral term [see also Eq. (9)].

Again, a recovery experiment with the EGM-96 gravity field model complete to degree and order 120 was conducted, this time including random non-conservative accelerations with a standard deviation of $10^{-6}$ $\mathrm{m} / \mathrm{s}^{2}$ and zero mean. The change in total energy, i.e. the sum of kinetic and potential energy, is displayed in Fig. 4, together with the dissipated energy obtained by solving Eq. (9). It must be noted that because a random time series of non-conservative forces was generated with zero mean, this may lead to both energy decreases and increases at a certain time instant, which can be clearly observed. It can also be seen that, as to be expected, the left and right part of this figure are mirror images, and the sum is equal to zero apart from numerical integration and computer round-off errors. In fact, the RMS of the sum was found to be equal to $0.0027 \mathrm{~m}^{2} / \mathrm{s}^{2}$ (Table 1), with minimum and maximum values of -0.0240 and $0.0215 \mathrm{~m}^{2} / \mathrm{s}^{2}$ with respect to the mean. The RMS is about equal to $\sqrt{2}$ times the numerical integration error for the pure gravitational orbit
(Sect. 4.2), a factor which can be expected when summing two uncorrelated error distributions.

The iterative procedure for gravity recovery complete to degree and order 120 again converged after seven iterations with a global geoid error RMS of $25 \mathrm{~mm}$ (6 $\mathrm{mm}$ excluding the polar gaps), which can be attributed to numerical integration error of both the orbit equations of motion and the dissipative term (Eqs. (9)], similarly as for the test case described in the previous section. Fluctuations similar to the test case without the random accelerations (previous section) could be observed for zonal gravity field terms for higher iterations. It can be concluded that observed non-conservative accelerations can be easily included in the iterative energy integral method.

\subsection{Sensitivity to orbit error}

As indicated in Sect. 2, the gravity field recovery method based on the energy integral is in fact a two-stage procedure, the first stage consisting of a precise orbit determination and the second stage of the gravity field parameter estimation. Although the focus of this paper is on the second stage, attention needs to be paid to the accuracy of the precise orbit determination, more specifically to the accuracy of the determined satellite positions and velocities. Based on Eq. (11), the sensitivity to position and velocity uncertainties in the ECI frame can be established. Taking into account first order terms only, the following relation can be derived:

$$
\begin{aligned}
\Delta(H+U)= & \left(\dot{x}_{i} \Delta \dot{x}_{i}+\dot{y}_{i} \Delta \dot{y}_{i}+\dot{z}_{i} \Delta \dot{z}_{i}\right) \\
& +\left(\dot{x}_{i} \omega_{e} \Delta y_{i}-\dot{y}_{i} \omega_{e} \Delta x_{i}\right)+\left(\omega_{e} y_{i} \Delta \dot{x}_{i}-\omega_{e} x_{i} \Delta \dot{y}_{i}\right)
\end{aligned}
$$

where, $\Delta(H+U)$ is the energy observation error, and $\Delta x_{i}, \Delta y_{i}, \Delta z_{i}$ and $\Delta \dot{x}_{i}, \Delta \dot{y}_{i}, \Delta \dot{z}_{i}$ denote the position and velocity errors, respectively. By analyzing this equation, it can be derived that position and velocity errors enter into the energy equation with different multiplication factors

$\mathcal{O}(\Delta(H+U))_{\text {pos }} \div \Delta P_{\text {sat }} V_{\text {sat }} \omega_{e}$

$$
\mathcal{O}(\Delta(H+U))_{\mathrm{vel}} \div \Delta V_{\mathrm{sat}} \sqrt{V_{\mathrm{sat}}^{2}+\omega_{e}^{2} P_{\mathrm{sat}}^{2}}
$$
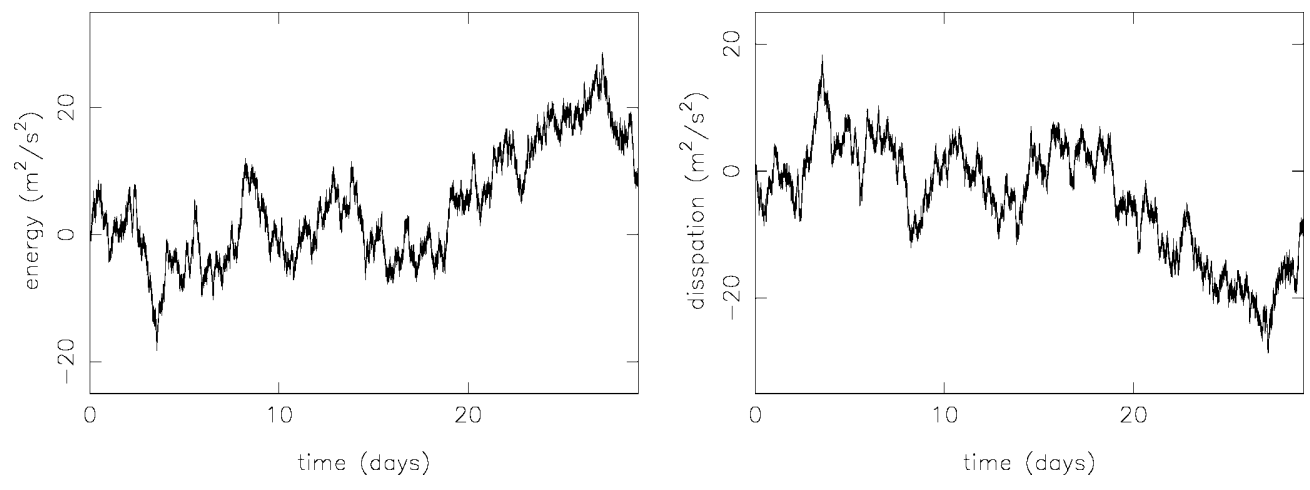

Fig. 4. Change of total energy of a GOCE-type orbit for a gravity field model complete to degree and order 120 and random accelerations with a standard deviation of $10^{-6} \mathrm{~m} / \mathrm{s}^{2}$ (left) and the dissipated energy (right) 
where $\mathcal{O}$ denotes order of magnitude, $V_{\text {sat }}$ is the absolute value of the satellite velocity $(\approx 7.7 \mathrm{~km} / \mathrm{s}$ for GOCE), $P_{\text {sat }}$ is the absolute value of the satellite position $(\approx 6630$ $\mathrm{km}$ for GOCE), and $\Delta P_{\text {sat }}$ and $\Delta V_{\text {sat }}$ are proportional to the orbit determination errors, or $\sqrt{\Delta x_{i}{ }^{2}+\Delta y_{i}{ }^{2}+\Delta z_{i}{ }^{2}}$ and $\sqrt{\Delta \dot{x}_{i}^{2}+\Delta \dot{y}_{i}^{2}+\Delta \dot{z}_{i}^{2}}$, respectively. The ratio $\rho_{\mathrm{obs}, \epsilon}$ between energy observation error due to position and velocity errors is thus proportional to

$\rho_{\text {obs }, \epsilon}=\frac{O(H+U)_{\text {err,pos }}}{O(H+U)_{\mathrm{err}, \mathrm{vel}}} \div \frac{\omega_{e} \Delta P_{\text {sat }}}{\Delta V_{\text {sat }} \sqrt{1+\frac{\omega_{e}{ }^{2} \text { sat }^{2}}{V_{\text {sat }}{ }^{2}}}}$

Typically, for classical dynamic orbit determination approaches, position errors cluster at 1 cycle per orbital revolution (cpr) and to a lesser extent at $0 \mathrm{cpr}$. In this case, the ratio has the following order of magnitude:

$\rho_{\mathrm{obs}, \epsilon}=\frac{\omega_{e}}{\omega_{\mathrm{sat}} \sqrt{1+\frac{\omega_{e}^{2}}{\omega_{\mathrm{sat}^{2}}{ }^{2}}}}$

For typical CHAMP, GRACE, and GOCE orbits, this ratio is between $1 / 15$ and $1 / 16$ since these satellites complete 15-16 orbital revolutions per day, which would indicate that the conversion of the orbit solution to energy observation is seriously hampered by velocity coordinate errors. However, the situation will change when adopting reduced-dynamic or kinematic orbit determination approaches. Due to the specific nature of these approaches, orbit error spectra might change drastically, leading to a more noisy behavior of position and velocity errors, although with smaller amplitudes for the position errors.

A gravity field recovery experiment was conducted in the presence of position and velocity errors coming from a precise orbit determination experiment described in detail in Visser and van den IJssel (2000, Table 4, $\left.\mathrm{CHAMP}^{c, d}\right)$. The orbit determination resembled a reduced-dynamic approach: empirical accelerations were estimated in $10 \mathrm{~min}$ intervals. This test case was extended to a period of 10 days, enabling a gravity field recovery experiment complete to degree and order 80 (as opposed to the recovery experiments in the previous sections, which were complete to degree and order 120). The RMS of inertial $x, y, z$ position and velocity errors is equal to $1.5,1.5,1.6 \mathrm{~cm}$, and $0.10,0.08,0.08 \mathrm{~mm} / \mathrm{s}$, respectively. In this case, the ratio $\rho_{\text {obs. } \epsilon}$ is equal to 0.013 or $1 / 78$, which is much worse than even the estimated guess for the dynamic orbit determination approach mentioned above. The latter can be explained by considering that for each 10 min empirical accelerations were estimated, which leads to a kind of averaging interval of $600 \mathrm{~s}$ when deriving for example, velocity from position estimates: position errors of a few centimeters then lead to velocity errors of the order of $0.1 \mathrm{~mm} / \mathrm{s}$.

Three different gravity field recovery experiments were conducted, taking into account (1) only position coordinate errors, (2) only velocity coordinate errors, and (3) both position and velocity coordinate errors. The last case is of course the most realistic one. The RMS of energy observation errors was found to be equal to $0.0058,0.6226$, and $0.6229 \mathrm{~m}^{2} / \mathrm{s}^{2}$ for the first, second, and third cases, respectively (Table 1). Thus the effect of velocity coordinate errors exceeds by far the effect of position coordinate errors. However, the amplitude spectra for the energy observation errors are quite different (Fig. 5). At low frequencies, in the bandwidth from $10^{-4}$ to $10^{-3} \mathrm{~Hz}$, position coordinate errors lead to a more or less flat spectrum, whereas the velocity coordinate errors lead to a rise in amplitude with increasing frequency. The latter can be explained by considering that velocity is the time derivative of position, leading to a stronger amplification at higher frequencies. In the bandwidth from $10^{-3}$ to about $5 \times 10^{-2}$ a strong decay can be observed for position coordinate errors and a more or less flat behavior for velocity coordinate errors. Finally, at even higher frequencies, a steep decline can be observed for the velocity coordinate errors, which is caused by the smoothing effect due to the estimation of empirical accelerations in the precise orbit determination (Visser and van den IJssel 2000).

The gravity field recovery performance in the presence of velocity errors (cases 2 and 3 ) is about two orders of magnitude worse compared to the performance in the presence of only position coordinate errors (case 1, Fig. 6). The recovery error in terms of global geoid RMS is equal to 58.5 and $0.4 \mathrm{~cm}$ for cases $2 / 3$ and case 1 , respectively. Due to the truncation at degree 80 , the polar gaps hardly play a role. Therefore, it is anticipated that the gravity field recovery errors will be much bigger when truncating at higher degrees, which may be suppressed to a certain extent by regularization. Based on these results, it can be concluded that velocity errors
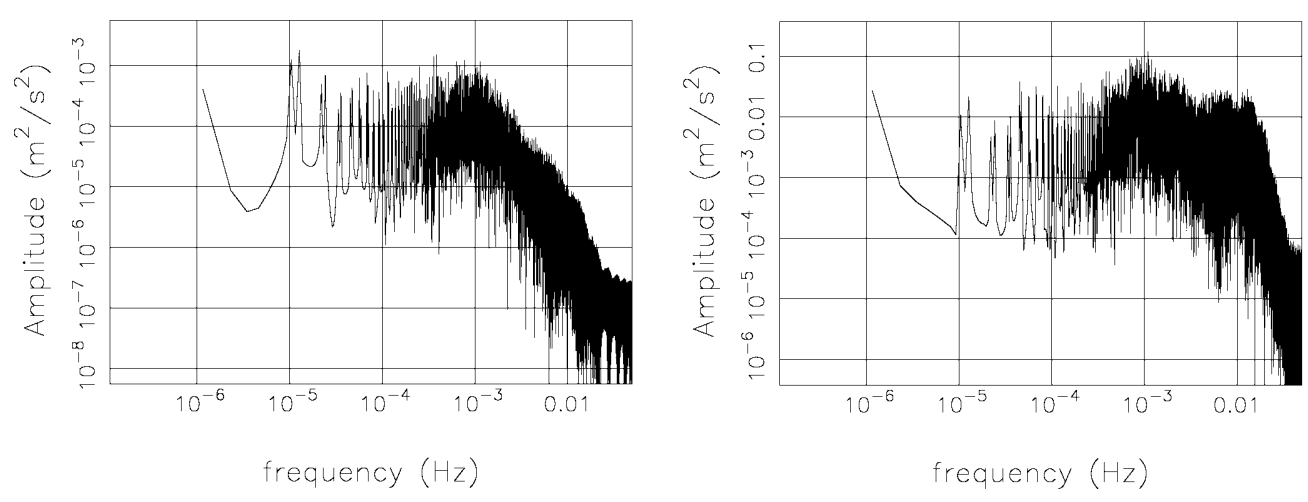

Fig. 5. Amplitude spectrum of energy observation errors due to position coordinate errors (left) and velocity coordinate errors (right) 

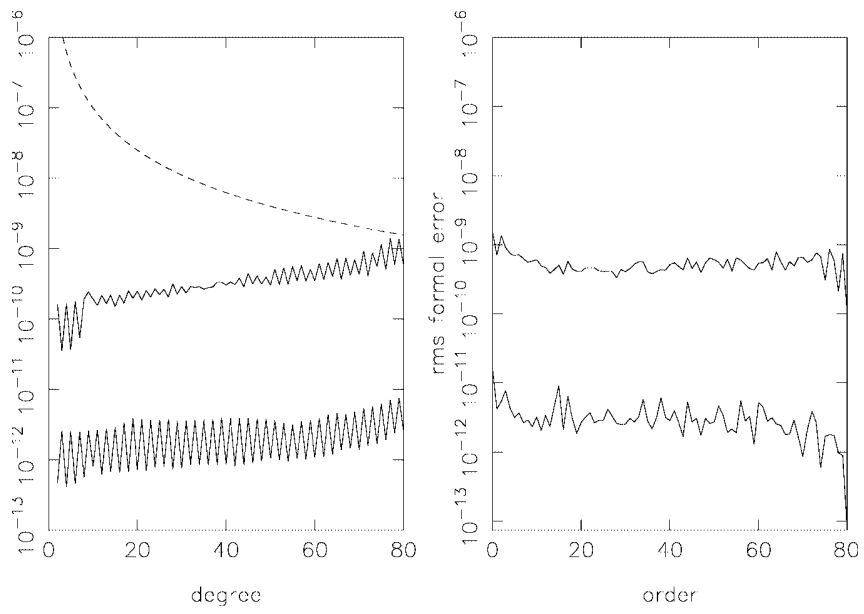

Fig. 6. Result of a gravity field recovery experiment complete to degree and order 80 based on a 10-day simulated data set of orbit perturbations with orbit determination errors included. The lower solid line (left and right) denotes the gravity recovery error when only the position errors are included after convergence (7th iteration). The upper solid line consists of two lines which cannot be distinguished. It represents the two cases where velocity errors are included

ruin the gravity field recovery performance when using energy equations. This will not be the case when using more classical approaches where the velocity coordinates enter the observation equations via a linear term, as opposed to a square term in energy equations, i.e. in the form of kinetic energy. Therefore, the question needs to be addressed how velocity errors can be reduced significantly when using methods based on energy equations. This can only be achieved by adopting a different orbit determination strategy. It has to be realized that, in principle, the primary product of a precise orbit determination is the satellite position coordinates, with the velocity coordinates as a derived, secondary, product.

The orbit determination is based on GPS pseudorange and carrier phase observations. The pseudo-range observations are absolute range measurements at about the $0.5-\mathrm{m}$ accuracy level and contain information for absolute positioning. The carrier phase observations are in fact range change observations at the few millimeter accuracy level and provide the information for reducing the positioning uncertainty down to the few centimeter level (ESA 1999; Visser and van den IJssel 2000). The GPS observing geometry is changing continuously, with certain GPS satellites rising and others setting. In a kinematic and reduced-dynamic precise orbit determination strategy, this easily leads to discontinuities in position coordinate errors at the centimeter level over short time spans. As shown above, this does not lead to serious errors in the energy observation equations. However, these discontinuities lead to velocity errors up to a few tenths of millimeter per second and result in large kinetic energy errors due to the squaring of the absolute velocity. It might be possible to improve the velocity estimates in an off-line process by selecting only continuous links between the GPS satellites and the user satellite within a moving window centered around the time for which the velocity is to be derived. For example, when using a normal point window of $5 \mathrm{~min}$ and a $1-\mathrm{Hz}$ sampling, the velocity of the user satellite can be determined with an accuracy of $0.006 \mathrm{~mm} / \mathrm{s}$, assuming an ionospheric-free carrier phase noise level of $9 \mathrm{~mm}$ and a second-order polynomial fit evaluated at the center of the window. With a typical geometric dilution of precision (GDOP) value of less than 3, this leads to an absolute velocity error of less than $0.018 \mathrm{~mm} / \mathrm{s}$, compared to the $0.15 \mathrm{~mm} / \mathrm{s} 3$-dimensional velocity error of the test case described above $\left(\sqrt{0.1^{2}+0.08^{2}+0.08^{2}} \mathrm{~mm} / \mathrm{s}\right)$, or a reduction by more than a factor of 8 . It must be noted that by generating normal points by, for example, a secondorder polynomial, high-frequency velocity coordinate signals are smoothed as well, posing a limit to the length of the window. These kinds of procedures are, however, beyond the scope of this paper and are left for further research. It is interesting to note that similar questions have been addressed in deriving accelerations in airborne gravimetry using GPS and Inertial Navigation Systems (Jekeli and Garcia 1997).

\section{Discussion and conclusions}

An efficient tool for fast gravity field recovery up to high degree and order from orbit perturbations has been developed and implemented successfully. The associated method, referred to as iterative energy integral method, was first verified by simulating a 29day test orbit in the presence of a $120 \times 120$ gravity field model. The block-diagonal approach required a total CPU time of $105 \mathrm{~min}$ (15 min per iteration) on a power PC (1.7 GHz clock speed), compared to 72 hours for computing the full matrix (1 iteration required), or a ratio of about 40 . For higher-degree spherical harmonic expansions, such as foreseen for GRACE and GOCE, this ratio will become much larger. In addition, the flexibility of the method with respect to measured non-conservative accelerations was proven by an experiment incorporating a time series of random accelerations. Remaining gravity field recovery errors could be attributed to numerical integration errors when solving the orbital equations of motion and when integrating the energy loss integral.

The sensitivity analysis in relation to orbit determination errors (for gravity field recovery complete to degree and order 80) revealed a serious sensitivity of the iterative energy integral method to velocity errors as opposed to a much reduced sensitivity to position errors. It must be noted that this sensitivity is not due to the iterative nature of this method, using a block-diagonal approximation of the normal matrix. Comparable results will be obtained when employing a full normal matrix approach. Therefore, special attention needs to be paid to strategies for improved recovery of velocities. A possibility to achieve this has been indicated and is a candidate for further research. Existing kinematic and reduced-dynamic orbit determination approaches more or less optimize the accuracy of position coordinates and yield the risk of relatively large velocity errors that can 
be attributed to noise amplification of the intrinsically position and position-change GPS observations.

Acknowledgments. The research described in this paper was for a large part triggered by a study carried out by the European GOCE Gravity Consortium (EGG-C) and supported by the European Space Agency (ESA).

\section{References}

Biancale R, Balmino G, Lemoine J-M, Marty J-C, Moynot B (2000), A new global Earth's gravity field model from satellite orbit perturbations: GRIM5-S1, Geophys Res Lett 27(22):3611-3614

Boyce WE, DiPrima RC (1986) Elementary differential equations and boundary value problems, 4 th edn. Wiley, New York

Colombo OL (1984) The global mapping of gravity with two satellites, vol 7, no. 3. Publications on Geodesy, New Series, Netherlands Geodetic Commission,

European Space Agency (1998) European views on dedicated gravity field missions: GRACE and GOCE. An Earth Sciences Division Consultation Document, ESD-MAG-REP-CON-001. European Space Agency, May

European Space Agency (1999) Gravity field and steady-state ocean circulation mission. Reports for mission selection, The four candidate earth explorer core missions, SP-1233(1). European Space Agency, July

Ilk, KH (1983) Formuliering von Energieaustauschbeziehungen zur Ausmessung des Gravitationfeldes. In: Schneider M (ed) Die Arbeiten des Sonderforschungsbereich 78 Satellitengeodäsie der Technische Universität München im Jahre 1982 Astronomisch-Geodätische Arbeiten der Bayerischen Kommission für die Internationale Erdmessung, no. 43

Jekeli C (1999) The determination of gravitational potential differences from satellite-to-satellite tracking. Celest Mech Dynam Astron 75:85-101

Jekeli C, Garcia R (1997) GPS phase accelerations for moving-base vector gravimetry, J. Geod 71(10):630-639

Kaula WM (1966) Theory of satellite geodesy. Blaisdell, Waltham, MA

Klees R, Koop R, Visser P, van den IJssel J (2000) Efficient gravity field recovery from GOCE gravity gradient observations. J. Geod 74(7/8):561-571

Lanczos C (1949) The variational principles of mechanics. University of Toronto Press, Toronto

Landau LD, Lifschitz EM (1965) Mechanik, Band I der Reihe Lehrbuch der Theoretischen Physik. Akademie-Verlag, Berlin

Lemoine FG, Smith DE, Kunz L, et al. (1997) The development of the NASA GSFC and NIMA Joint Geopotential Model. In: International Association of Geodesy Symposia, Gravity,
Geoid and Marine Geodesy, vol, 117. Springer, Berlin Heidelberg New York, pp 461-469

Montenbruck O, Gill E (2000) Satellite orbits - models methods applications. Springer, Berlin Heidelberg New York

O'Keefe JA (1957) An application of Jacobi's integral to the motion of an Earth satellite. Astron J 62 (1252):265-266

Reigber C (1969) Zur Bestimmung des Gravitationfeldes der Erde aus Satellitenbeobachtungen. In: Deutsche Geodätische Kommission, Reihe C, no. 137

Reigber Ch, Schwintzer P, Lühr H (1999), The CHAMP geopotential mission. In: Marson I, Sünkel H (eds) Proc 2nd Joint Meeting of the International Gravity and the International Geoid Commission, Trieste 7-12 September. 1998. Boll Geofis Teor Appli 40(3-4):285-289

Reigber Ch, Schwintzer P, Koenig R, Neumayer K-H, Bode A, Barthelmes F, Foerste Ch, Balmino G, Biancale R, Lemoine JM, Loyer S, Perosanz F (2001) Earth Gravity Field Solutions from Several Months of CHAMP Satellite Data. EOS Trans. AGU Fall Meet. Suppl. 82(47):G4IC-02

Rowlands D, Marshall JA, McCarthy J, Moore D, Pavlis D, Rowton S, Luthcke S, Tsaoussi L (1995) GEODYN II system description, vols 1-5. Contractor rep, Hughes STX Corp, Greenbelt, MD

Rummel R, Colombo OL (1985) Gravity field determination from satellite gradiometry. Bull Geod 59:233-246

SID (2000) GOCE end-to-end closed loop simulation. Final report, ESTEC Contract no. 12735/98/NL/GD, Space Research Organization Netherlands, Institut für Gstronomische und Physikolische Geodásie, Delft Institute for Earth-Oriented Space Research

Sneeuw N, van Gelderen M (1997) The polar Gap. In: Sanco F, Rummel R (eds) Geodetic boundary value problems in view of the one centimeter geoid. Lecture Notes in Earth Sciences 65, pp 559-568, Springer

Tapley BD, Reigber C (1999) GRACE: a satellite-to-satellite tracking geopotential mapping mission. In: Marson I, Sünkel H (eds) Proc 2nd Joint Meeting of the International Gravity and the International Geoid Commission, Trieste, 7-12 September 1998. Boll Geofis Teor Appli 40(3-4):291.

Visser PNAM (1992) The use of satellites in gravity field determination and model adjustment. $\mathrm{PhD}$ dissertation, University. of Delft

Visser PNAM, van den IJssel J (2000) GPS-based precise orbit determination of the very low Earth orbiting gravity mission GOCE. J. Geod 74(7/8):590-602

Visser PNAM, Wakker KF, Ambrosius BAC (1994) Global gravity field recovery from the ARISTOTELES satellite mission. J Geophys Res 99(B2):2841-2851

Visser PNAM, van den IJssel J, Koop R, Klees R (2001) Exploring gravity field determination from orbit perturbations of the European Gravity Mission GOCE. J Geod 75(2/3):89-98 